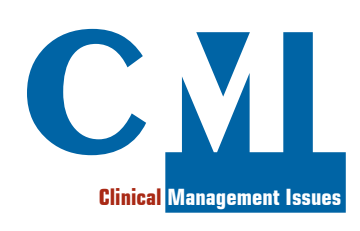

\title{
Atypical Presentation of Periodic Paralysis: A Case Report
}

\begin{abstract}
Hypokalemic periodic paralysis (HPP) is a kind of periodic paralysis, which is a heterogeneous group of muscle diseases. It is characterized by episodes of flaccid and sudden muscle weakness. Here, we present a case of HPP. The patient was referred to our department because of severe dizziness and fall. After a comprehensive evaluation, a markedly low potassium was detected. The patient's symptoms resolved after replacement of potassium and he was discharged without deficits. Even though the literature reports an association with exercise, carbohydrate load, and stress, further workup in our patient revealed no association with these precipitants. A proper differential diagnosis should rule out other causes of weakness and paralysis, thus allowing a timely treatment.
\end{abstract}

Keywords: Periodic Paralysis; Potassium; Muscle Weakness; Dizziness; Hypokalemia CMI 2019; 13(1): 7-10

https://doi.org/10.7175/cmi.v13i1.1383
1 Prof. Dr. Ilhan Varank Sancaktepe Training and Research Hospital, Department of Internal Medicine, Istanbul, Turkey

\section{INTRODUCTION}

Periodic paralyses (PP) are a heterogeneous group of rare inherited muscle disorders characterized by recurrent attacks of intermittent skeletal muscle weakness. They generally affect limbs and, even though they resolve spontaneously, over time they may result in permanent miopathy.

During attacks, changes in anion channels occur, particularly in potassium channels [1]. PP may be hypokalemic, hyperkalemic, or normokalemic.

Hypokalemic periodic paralysis (HPP) is characterized by low levels of potassium during attacks, even though not necessarily below normal range.

The genes affected are generally those encoding for a calcium channel (chromosome 1) or a sodium channel (chromosome 17) [2]. The therapy generally consists in the administration of potassium during the attacks, while avoiding intense effort and carbohydrate-rich meals is useful as prophylaxis.
A HPP may be caused also by thyrotoxicosis and potassium loss due to gastrointestinal and renal diseases [2].

\section{CASE DESCRIPTION}

A 42-year-old male came to the Emergency Room complaining for a sudden onset of severe dizziness and fall. The anamnesis did not reveal any significant pre-existing conditions.

He had no dizziness or weakness neither the day before, nor that very morning. At

Why Do we Describe This Case

Dizziness and weakness are common symptoms and may be caused by several illnesses. Hypokalemic periodic paralysis is a rare disease, but should be taken into consideration after a proper diagnostic workup
Corresponding author Aysun Isiklar

Prof. Dr. Ilhan Varank Sancaktepe Training and Research Hospital, Department of Internal Medicine Emek neighborhood, Namik Kemal St. N. 54 34785 Sancaktepe/İstanbul Tel: 00902166063300 Fax: (0216) 6063395 aysunisiklar@gmail.com

Received: 15 November 2018 Accepted: 25 January 2019 Published: 4 February 2019 
Figure 1. Timeline of interventions and outcomes

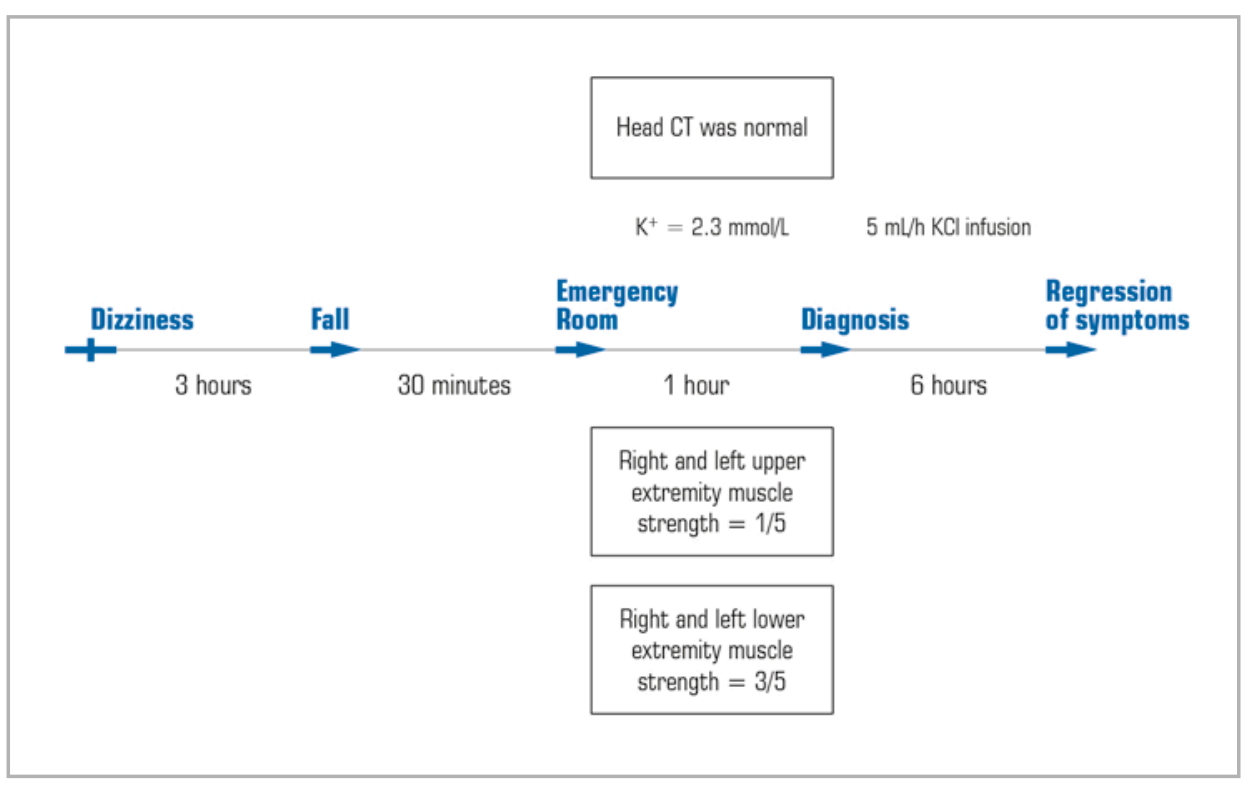

noon time he felt a mild dizziness, which had been increasing in the following 2 hours, when he fell at home as a result of the sudden onset of muscle weakness. The dizziness was described as motion of the environment, while the weakness was bilateral and involved both the proximal muscles of the shoulders and hips as well as the distal extremities. He had no respiratory and swallowing difficulties and was able to move his neck and face muscles. He denied any pain, paresthesia, diarrhea, shortness of breath, or weight loss in the preceding period. His parents and brothers had no history of similar episodes and no other significant illnesses.

The physical examination revealed that he was conscious and cooperated. Arterial blood pressure was $130 / 80 \mathrm{mmHg}$ and heart rate was 80 beats per minute. His weight was normal. His skin was cold and dry, and the oral mucosa was moist. No jugular venous distension, goiter, or lymphadenopathy were detected.

Cardiovascular examination revealed a normal heart rate, with a regular rhythm, and no murmurs. Proper examinations revealed that lungs and abdomen were unremarkable. There were neither deformity nor edema in the extremities, while distal pulses were bilateral and equal. At neurological examination, his right and left upper extremity muscle strength was $1 / 5$ and his right and left lower extremity muscle strength was $3 / 5$. He had rotatory nystagmus. Proximal tendon reflexes were decreased and sensation was intact.

Blood tests, liver enzymes, and complete blood count were normal, except for a po- tassium level of $2.3 \mathrm{mmol} / \mathrm{L}$ (normal range: 3.5-5.1 mmol/L). In particular, in addition to low potassium, arterial blood gas revealed $\mathrm{pH}=7.4$ and $\mathrm{HCO}_{3}{ }^{-}=22 \mathrm{mmol} / \mathrm{L}$. Urinary potassium rate was in normal range and creatine phosphokinase (CPK) level moderately raised during the attack. The compound muscle action potential (CMAP) amplitude declined during the paralytic attack. Cardiac evaluation was provided with electrocardiogram (ECG): hypokalemia presented as flat $T$ waves. The patient, who was thought to be affected by conversion disorder, underwent neurological consultation for the differential diagnosis. Dizziness was due to benign paroxysmal positional vertigo, and on Dix-Hallpike maneuver classic rotatory nystagmus with latency and limited duration was considered pathognomonic. Other cranial nerve function was intact. Computed tomography of brain was performed to determine the etiology of the fall and was considered to be normal.

Six hours after initiation of potassium infusion $(\mathrm{KCl}[2 \mathrm{mEq} / \mathrm{mL}])$ at $5 \mathrm{ml} / \mathrm{h}$ through a peripheral vein, the patient's neurological symptoms had completely resolved. Further investigation were performed to elucidate the etiology of hypokalemia. Urine sodium and potassium, and serum aldosterone and renin levels were measured to rule out adrenal involvement and were found to be normal. Thyroid stimulating hormone was normal (Figure 1).

The patient was diagnosed with hypokalemic periodic paralysis with unknown etiology and started a treatment with potassiumsparing diuretic. He was discharged, but a 
follow-up visit with an endocrinologist was planned.

\section{DISCUSSION}

"Dizziness" is a nonspecific term commonly used by patients to identify symptoms. It includes vertigo, nonspecific dizziness, disequilibrium, and presyncope. The first step in the evaluation is to properly categorize the patient.

Weakness is a common, nonspecific, presentation in both the emergency and outpatient settings. Therefore, the differential diagnosis for dizziness and weakness is extensive. First of all, strokes and tumors that cause nerve entrapment are potentially lifethreatening and should be ruled out.

For the diagnosis of HPP, the duration and distribution of symptoms should be evaluated together with a detailed anamnesis. Generally, this disease is not taken into account during the first run.

The patient was diagnosed with HPP, whose prevalence rate was thought to be approximately 1:100,000 in further consideration [3].HPP is a disorder affecting more commonly males with ages ranging between 20 and 40 years. The clinical features of the syndrome vary slightly depending on the underlying etiology. Although the serum potassium level is often alarmingly low, other electrolytes are usually normal [4]. However, the total body potassium is actually normal, with the change in the serum level reflecting a shift of potassium into cells. Between paralytic attacks, muscle strength and potassium levels are normal, thus diagnosis at this period is difficult. Electrocardiographic changes are common, but differ from patients with true low potassium. Electromyography reveals abnormalities in some patients, but is usually normal between episodes, when there is no clinically detectable weakness.

HPP may be due to various causes, thus extensive research may be required in order to elucidate the underlying etiology. HPP may occur sporadically, spontaneously or as the result of autosomal dominant inheritance. This form shows a deterioration of cellular potassium regulation due to abnormalities in sodium or calcium channels. Acute paralytic attacks are treated with potassium replacement. Cardiac rhythm and serum potassium levels should be closely monitored.
Diagnostic criteria for primary hypokalemic periodic paralysis have been published by a Cochrane review [6]. Diagnosis without genetic analysis, according to one of these criteria, can be made, as in this patient, by the detection of recurrent attacks with muscle weakness with documented serum $\mathrm{K}^{+}<2.5 \mathrm{mEq} / \mathrm{L}$. The patient was also consistent with the other three diagnostic criteria for hypokalemic periodic paralysis: duration of paralysis longer than two hours, improvement in paralysis symptoms with potassium replacement, and recurrence of symptoms under the certain periods and circumstances $[5,6]$.

However, diarrhea, chest pain, shortness of breath, or weight loss, that sometimes precede the onset of HPP symptoms, were absent in this patient.

HPP can be triggered by the effect of factors such as alcohol, anesthesia, excessive consumption of carbohydrate-rich meal, stress, insulin, strenuous exercise, and steroids. In rare cases, cold or overtemperature seemed to induce paralysis at regular intervals, but data are too scarce. In general, after exclusion of the other causes, the main treatment is potassium replacement. The remaining supportive treatment is correction or avoidance of the other triggering factors. Hypokalemia occurs before the onset of symptoms, thus precautions can be taken with the previously mentioned close followup. Normal saline $(0.9 \%)$ should be preferred, while dextrose-containing solutions should be avoided during fluid replacement.

Symptoms such as sweating, myalgia, mental dullness, irritability, and palpitations also occur rather than muscle weakness during the attacks [7].

Several previously reported cases of HPP concern children [8], and most of them are associated with thyrotoxicosis $[9,10]$.

This case was not completely consistent with the other patients with regards to age and triggering factors, but he was considered to be HPP after differential diagnosis and in consequence of the response to the treatment performed. Although the patient was planned to be discharged upon obtaining a potassium level of $3.5 \mathrm{mmol} / 1, \mathrm{a} \mathrm{K}{ }^{+}$rich and $\mathrm{Na}^{+}$poor diet was recommended in the long term since he experienced the second attack. The patient was informed regarding the triggering and alarming symptoms.

It should be kept in mind that even though age and triggering factors do not always overlap, HPP should be considered. 


\section{Key Points}

- Dizziness and weakness are common symptoms

- Hypokalemic periodic paralysis (HPP) is characterized by episodes of flaccid and sudden muscle weakness

- Generally, it affects males with ages ranging between 20 and 40 years

- Serum potassium level is often alarmingly low, but other electrolytes are usually normal

- Acute paralytic attacks are treated with potassium replacement

- Cardiac rhythm and serum potassium levels should be closely monitored

- HPP may be due to various causes, thus extensive research may be required in order to elucidate the underlying etiology

- Among the triggering factors, there are alcohol, anesthesia, excessive consumption of carbohydrate-rich meal, stress, insulin, strenuous exercise, and steroids

\section{Funding}

This article has been published without the support of sponsors.

\section{Conflicts of Interests}

The author declares she has not competing financial interests concerning the topics of this article.

\section{REFERENCES}

1. Zacchia M, Abategiovanni ML, Stratigis S, et al. Potassium: From Physiology to Clinical Implications. Kidney Dis (Basel) 2016; 2: 72-9; https://doi.org/10.1159/000446268

2. Giordana MT, Calvo A, Canosa A. Segni e sintomi inusuali o rari in Neurologia. Torino: SEEd Medical Publishers, 2018

3. Abbas H, Kothari N, Bogra J. Hypokalemic periodic paralysis. Natl J Maxillofac Surg 2012; 3: 220-1; https://doi.org/10.4103/0975-5950.111391

4. Lin SH, Lin YF, Chen DT, et al. Laboratory tests to determine the cause of hypokalemia and paralysis. Arch Intern Med 2004; 164: 1561-6; https://doi.org/10.1001/archinte.164.14.1561

5. Vicart S, Sternberg D, Arzel-Hézode M, et al. Hypokalemic Periodic Paralysis. 2002 Apr 30 [Updated 2014 Jul 31]. In: Adam MP, Ardinger HH, Pagon RA, et al. (editors). GeneReviews ${ }^{\circledR}$ [Internet]. Seattle (WA): University of Washington, Seattle, 1993-2018

6. Sansone V, Meola G, Links TP, et al. Treatment for periodic paralysis. Cocbrane Database Syst Rev 2008; (1): CD005045; https://doi.org/10.1002/14651858.CD005045.pub2

7. Frey K, Holman S, Mikat-Stevens M, et al. The recovery profile of hyperbaric spinal anesthesia with lidocaine, tetracaine, and bupivacaine. Reg Anesth Pain Med 1998; 23: 159-63; https://doi. org/10.1097/00115550-199823020-00008

8. Mosquera Gorostidi A, ZarikianDenis SE, Gembero Arroyo E, et al. Hypokalemic periodic paralysis: A case report based on clinical and genetic findings. Eur J Paediatr Neurol 2015; 19 (Suppl 1): S126; https://doi.org/10.1016/S1090-3798(15)30427-X

9. Kelley DE, Gharib H, Kennedy FP, et al. Thyrotoxic periodic paralysis. Report of 10 cases and review of electromyographic findings. Arch Intern Med 1989; 149: 2597-600; https://doi. org/10.1001/archinte.1989.00390110139031; https://doi.org/10.1001/archinte.149.11.2597

10. Soule BR, Simone NL. Hypokalemic Periodic Paralysis: a case report and review of the literature. Cases J 2008; 1: 256; https://doi.org/10.1186/1757-1626-1-256 